\title{
ÉTUDE DE LA VALEUR NUTRITIONNELLE DE LA LEVURE UTILISÉE SOUS TROIS FORMES DIFFÉRENTES : LEVURE SÈCHE, LEVURE AUTOLYSÉE ET AUTOLYSAT DE LEVURE
}

\author{
C. CALET, J. ADRIAN et R. JACQUOT. \\ avec la collaboration technique de R. Helias-Frangne. \\ Station de Recherches aricoles, \\ Centre national de Recherches zootechniques, Jouty-en-Josas (Seine-et-Oise); \\ Centre de Recherches sur la Nutrition, \\ Centre national de la Recherche scientifique, Bellevue (Scine-et-Oise)
}

\section{SOMMAIRE}

Plusieurs études ont été poursuivies à la fois chez le Rat et chez le Poussin a fin de comparer l'efficacité de la levure présentée dans l'aliment soit sous forme de levure sèche, soit sous forme de levure autolysée, soit enfin sous forme d'autolysat de levure. Nous avons recherché en outre dans quelle mesure l'autolysat augmente la disponibilité des principes alimentaires contenus dans la levure, en particulier en ce qui regarde la méthionine.

Nous nous sommes adressés à des levures de bière et dans le cas du Rat, nous avons étudié également la levurc cultivée sur lactosérum.

Nos résultats montrent que :

$I^{0}$ Les deux types de levure sont susceptibles de valoriser un régime constitué de protides d'origine végétale (arachide, gluten) lorsque l'on effectue une substitution isoazotée.

$2^{0}$ I'autolysat de levure permet une croissance plus rapide que la levure originelle.

$3^{0}$ Toutefois cette amélioration n'est qu'apparente car elle porte essenticllement sur une augmentation des ingérés. L'indice de consommation et le coefficient d'efficacité protidique sont rigoureusement les mêmes dans les deux cas. L'autolysat agit essentiellement comme un facteur d'appétence intéressant.

$4^{\circ}$ Avec un régime déficient en lysine (cas du Rat) l'addition de méthionine à la levure ne provoque aucun avantage quels que soient le traitement et l'origine de la levure considérée. Avec un régime équilibré (cas du Poussin) la levure est valorisée par l'adjonction de méthionine mais le bénéfice est égal avec les trois formes de levure. Il semble donc que la méthionine soit également disponible dans la levure sèche ou autolysée et que le simple séchage de la levure sur cylindres soit suffisant pour rendre complète l'utilisation de cet acide aminé.

\section{INTRODUCTION}

La forme sous laquelle les principes nutritifs sont présentés dans l'aliment retentit directement sur leur utilisation. Bien que l'analyse révèle leur présence, ils ne sont pas pour autant " disponibles " pour l'animal; ils le deviennent cependant 
lorsque l'aliment a été prédigéré plus ou moins profondément et réduit en nutriments: résorbables.

Les méthodes qui réduisent une substance en ses éléments constitutifs ne sont pas toutes satisfaisantes. L'hydrolyse chimique est souvent trop violente et entraîne alors la destruction de certains aminoacides. L'hydrolyse enzymatique, moins brutale, libère les nutriments azotés en maintenant leur intégrité. C'est le cas des autolyses.

La levure est un aliment recherché pour sa teneur élevée en vitamines du groupe B. Elle présenterait un intérêt encore plus marqué pour l'alimentation des animaux si l'on pouvait mettre à profit sa richesse en lysine que limite cependant une déficience marquée en méthionine. Des expériences antérieures (JACQUOT I946) semblent indiquer que la disponibilité des acides aminés varie avec les modalités de préparation des levures : une levure séchée à basse température présente une valeur nutritionnelle plus faible qu'une levure traitée à température élevée. On sait également que pour être efficace la levure doit être tuée. On augmente considérablement sa valeur alimentaire lorsqu'on la soumet à un "éclatement " par chauffage : les membranes cellulaires sont ainsi rompues, livrant le cytoplasme à l'attaque des sucs digestifs.

Ce traitement bénéfique n'est peut-être pas suffisant pour fournir à la levure son efficacité maximum. On connaît en effet, d'autres conséquences du chauffage qui ne sont pas d'ordre physique mais d'ordre chimique et qui limitent la valeur nutritionnelle d'un aliment. On a cité de nombreux cas où les traitements thermiques rendent plus ou moins indisponibles les acides aminés constitutifs des aliments même si l'analyse révèle leur présence. Dans le cas de la levure soumise à haute température au cours du séchage, le cytoplasme est certes libéré mais il peut avoir subi des dommages et plus spécialement une altération de ses constituants azotés. On peut dès lors se demander si une attaque enzymatique douce telle que celle qui se produit au cours de l'autolyse de la levure séchée ne rehausse pas sa valeur alimentaire en rendant disponible l'ensemble des constituants des matières azotées. Aussi avons-nous recherché dans quelle mesure différents procédés d'autolyse ont une influence bénéfique sur l'efficacité de la levure pour la croissance des animaux.

Nous rapportons les résultats d'essais entrepris à la fois sur le Rat et sur le Poussin afin de comparer l'efficacité de trois produits: la levure sèche, la levure autolysée et l'autolysat de levure. Le premier échantillon correspond à une levure déshydratée sur cylindres (levure sèche). Le second se réfère à une levure autolysée obtenue par autodigestion. L'autolyse se poursuit spontanément sous antiseptiques dans des conditions de température $\left(5^{\circ}\right)$ et de $\mathrm{pH}$ convenables. On estime qu'elle est presque complète après 48 heures. Le produit ainsi obtenu est alors séché dans sa totalité. Le troisième échantillon est un autolysat de levure ainsi préparé : la levure autolysée est soumise à un essorage à grande vitesse qui assure la séparation entre les éléments solubles correspondant au contenu des cellules et les membranes cellulaires. La fraction soluble est ensuite concentrée sous vide de façon à obtenir un prođuit pâteux ne dépassant pas $3^{\circ} \mathrm{p}$. roo d'humidité. En bref, la levure autolysée renferme tous les constituants de la levure y compris la cellulose. On peut admettre que les substances azotées qu'elle renferme sont sous une forme disponible, alors qu'il n'en est pas de même dans la levure sèche. L'autolysat contient les éléments solubles de la levure autolysée dont il diffère par l'absence de cellulose. 


\section{MATÉRIEL, ET MÉTHODES}

Plusieurs expériences ont été poursuivies chez le Rat et chez le Poussin pour comparer l'efficacité de la levure de bière sous les trois formes précitées afin de préciser le bénéfice éventuel que la levure tire de l'autolyse en ce qui concerne la disponibilité de sa méthionine. La composition des produits utilisés est donnée dans le tableau $\mathrm{r}$.

TABLEAU I

Composition chimique de la levure de biere sèche et de son autolysat

\begin{tabular}{|c|c|c|}
\hline & Levure & Autolysat \\
\hline Humidité .................. & 8,4 & 29,2 \\
\hline Matière sèche ..... & 91,6 & 70,8 \\
\hline Matières minérales ............ & 6,9 & 7,1 \\
\hline Matières cellulosiques ............. & 5,7 & 0,3 \\
\hline Matières azotées totales ............ & 43,0 & 42,8 \\
\hline Matières azotées précipitables au cuivre & 36,8 & 17,6 \\
\hline Matières grasses $\ldots \ldots \ldots \ldots \ldots \ldots$ & 6,2 & 1,7 \\
\hline Phosphore total..$\ldots \ldots \ldots \ldots \ldots \ldots$ & 1,7 & 0,3 \\
\hline
\end{tabular}

On remarque un avantage certain de l'autolysat sur la levure en ce qui regarde la teneur en cellulose. Il est moins riche cependant en lipides et surtout en phosphore. L'abaissement marqué de l'azote précipitable au cuivre rend compte de la dégradation des formes protéiques complexes et de leur transformation en produits plus simples (acides aminés libres).

Chez le Rat une levure d'une autre origine, cultivée sur lactosérum, a été étudiée dans les mêmes conditions expérimentales. Nous ne possédons malheureusement pas les analyses de ce produit.

\section{ESSAI SUR POUSSIN}

Les poussins mâles issus de croisement Rhode $\times$ Wyandotte sont répartis en lots homogènes à l'âge de 8 jours. L'effectif de chaque lot est de 25 poussins. Ils sont élevés pendant 8 semaines en batteries et reçoivent l'eau et la nourriture à volonté.

La composition des régimes de base est donnée dans le tableau 2.

TABLEAU 2

Composition des régimes de base des poussins

\begin{tabular}{|c|c|c|c|}
\hline & R. B. 1 & R. B. & 2 \\
\hline Blé $\ldots \ldots \ldots \ldots \ldots \ldots \ldots \ldots \ldots$ & 16,0 & 32,0 & \\
\hline 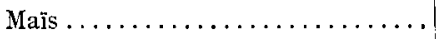 & 24,0 & 15,0 & \\
\hline Orge $\ldots \ldots \ldots \ldots \ldots \ldots \ldots \ldots$ & 9,0 & 10,0 & \\
\hline Avoine décortiquée $\ldots \ldots \ldots \ldots \ldots$ & - & 5,0 & \\
\hline Farine de luzerne déshydratée...... & 3,0 & 3,0 & \\
\hline Tourteau d'arachide ............. & 12,5 & 15,0 & \\
\hline Tourteau de soja cuit .......... & 8,5 & - & \\
\hline Tourteau de sésame $\ldots \ldots \ldots \ldots$ & & 5,0 & \\
\hline Poudre de lait écrémé $\ldots \ldots \ldots \ldots$ & 2,5 & 2,0 & \\
\hline Farine de poisson $\ldots \ldots \ldots \ldots \ldots$ & 3,0 & - & \\
\hline 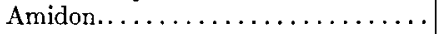 & 8,0 & 4,0 & \\
\hline Poudre d'os ................ & 2,0 & 1,5 & \\
\hline $\mathrm{CO}_{3} \mathrm{Ca} \ldots \ldots \ldots \ldots \ldots \ldots \ldots \ldots$ & 1,0 & 1,0 & \\
\hline Chlorure de sodium $\ldots \ldots \ldots \ldots \ldots$ & 0,4 & 0,5 & \\
\hline Oligosels $\ldots \ldots \ldots \ldots \ldots \ldots \ldots \ldots$ & 0,5 & 0,5 & \\
\hline Complément vitaminique $\ldots . . . \ldots$. & 0,5 & 0,5 & \\
\hline DL méthionine $\ldots \ldots \ldots \ldots \ldots$ & 0,1 & - & \\
\hline Total $\ldots \ldots \ldots \ldots \ldots \ldots \ldots \ldots$ & 92,0 & 95,0 & \\
\hline
\end{tabular}


Le complément vitaminique apporte par Ioo kg de régime 800000 U. I. de vitamine $A$, 100000 U. I. vitamine $\mathrm{D}_{3}$, , $00 \mathrm{mg}$ de riboflavine et $5 \circ \mathrm{g}$ de choline.

Le régime de base $\mathrm{R} B$ I est complet et équilibré. Il est distribué dans les périodes expérimenles selon le protocole résumé dans le tableau 3.

TABLEAU 3

Schéma du protocole expérimental R. B. 1

\begin{tabular}{c|c|c|c|c}
\hline Lots & $\begin{array}{c}\text { Régime de base } \mathrm{n}^{\circ} \\
\%\end{array}$ & $\begin{array}{c}\text { Levure } \\
\%\end{array}$ & $\begin{array}{c}\text { Autolysat de levure } \\
\%\end{array}$ & $\begin{array}{c}\text { Tourteau d'arachide } \\
\%\end{array}$ \\
\hline I & 92 & 0 & - & 8 \\
II & 92 & 6 & - & 2 \\
III & 92 & - & 2 & - \\
\hline
\end{tabular}

Les régimes sont isoazotés avec un taux protéique $(\mathrm{N} \times 6,25)$ de $20,6 \mathrm{p}$. cent car la levure, l'autolysat de levure et le tourteau d'arachide possèdent la même teneur azotée (43 p. Ioo).

Le régime de base $\mathrm{R} \mathrm{B} 2$, avec un taux protéique de 18,4 p. 100 et 2260 calories productives par $\mathrm{kg}$ (FRAPS, 1946), est principalement carencé en lysine et en méthionine. L'apport de 5 p. Ioo de levure comble le déficit du premier acide aminé mais accuse celui du second. D'après BALDini et ROSENBERG, I955, le besoin du poussin en méthionine est de o, $5^{\mathrm{I}} \mathrm{p}$. Ioo du régime pour la valeur énergétique considérée. Notre régime n'apporte que 0,2 p. Ioo de méthionine ce qui fait apparaitre un déficit de 73 p. xoo pour cet acide aminé. Afin de mettre en évidence le bénéfice éventuel que la levure tire du mode de préparation nous avons comparé les efficacités de la levure entière et de la levure autolysée en présence ou non d'un supplément de méthionine.

Le protocole utilisé avec le régime $\mathrm{R} \mathrm{B} 2$ est donné dans le tableau 4.

TABLEAU 4

Schéma du protocole expérimental R. B. 2

\begin{tabular}{|c|c|c|c|c|c|c|}
\hline Lots & $\begin{array}{l}\text { Régime de } \\
\text { base } n^{\circ} 2\end{array}$ & $\begin{array}{l}\text { Lysine } \\
\text { DL }\end{array}$ & $\begin{array}{l}\text { Méthionine } \\
\text { DL }\end{array}$ & $\begin{array}{l}\text { Levure } \\
\text { entière }\end{array}$ & $\begin{array}{c}\text { Levure } \\
\text { autolysée }\end{array}$ & $\underset{\text { Gluten }}{\text { Amidon }}+$ \\
\hline V & 95 & - & - & - & - & $5 \%$ \\
\hline VI .. & 95 & 0,2 & 0,2 & - & - & $4,6 \%$ \\
\hline VII $\ldots$. & 95 & - & - & 5 & - & - \\
\hline VIII $\ldots$ & 94,8 & - & 0,2 & 5 & - & - \\
\hline XI $\ldots .$. & 95 & - & - & - & 5 & - \\
\hline X...... & 94,8 & - & 0,2 & - & 5 & - \\
\hline
\end{tabular}

Le lot témoin (lot VI) est supplémenté par de la lysine pure en quantité égale à celle apportée par 5 p. roo de levure. Tous les régimes étudiés, sauf celui du lot $V$, possèdent le même taux de lysine. Ils diffèrent théoriquement par le degré de disponibilité de leurs acides aminés et par leur teneur en méthionine.

\section{ESSAI SUR RAT}

96 rats mâles, pris au sevrage, sont répartis en i 2 lots homogènes.

L'expérimentation dure 80 jours pour les lots qui reçoivent la levure de bière et 46 jours pour les lots qui reçoivent la levure de lactosérum.

Ces produits, donnés en complément de protides d'origine végétale sont utilisés, soit seuls, soit supplémentés par de la méthionine. La ration de base, dont la composition figure dans le tableau 5 , apporte 14 p. Ioo de matières azotées totales $(N \times 6,25)$ dont la moitié provient de l'arachide et l'autre moitié de gluten de blé. 
TABLEAU 5

Composition du régime de base des rats (en p. cent)

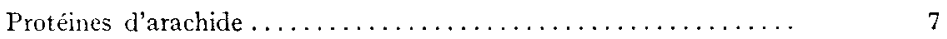

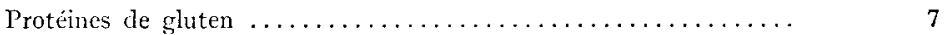

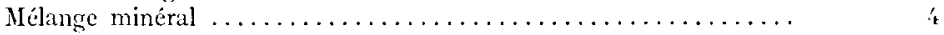

Mélange vitaminique. ......................... 1

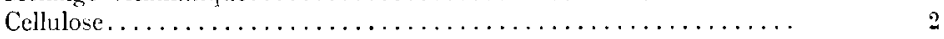

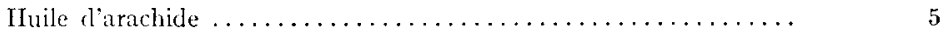

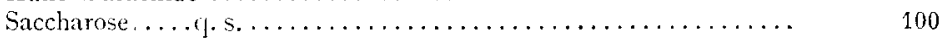

Les animaux reçoivent les régimes cxpérimentaux selon le schéma qui figure dans le tableau 6.

TABI,EAU 6

Schimat du protocole expírimental

\begin{tabular}{|c|c|c|}
\hline $\begin{array}{c}\text { Substitution aux protéines du régime } \\
\text { de base }\end{array}$ & $\begin{array}{l}\text { Lots recevant } \\
\text { la Levure de Bière }\end{array}$ & $\begin{array}{l}\text { Lots recevant la } \\
\text { Levure de Lactosírum }\end{array}$ \\
\hline risu & $A$ & $A^{\prime}$ \\
\hline 4. p 100 protéines de levure sèche . . . . . . & $\mathrm{B}$ & $B^{\prime}$ \\
\hline 1. 100 protéines de levure autolysée . . & $\mathrm{C}$ & $C^{\prime}$ \\
\hline 4. 100 yrotéines de autolysat de levure & $\mathrm{D}$ & 一 \\
\hline Lot $\mathrm{B}+0,1 \mathrm{p} .100 \mathrm{de} \mathrm{DI}$, méthionine $\ldots \ldots$ & $\mathrm{E}$ & $E^{\prime}$ \\
\hline Lot $\mathrm{C}+0,1 \mathrm{p}, 100$ de $\mathrm{DL}$ méthionine ..... & $\mathrm{F}$ & $F^{\prime}$ \\
\hline Lot $\mathrm{D}+0,1 \mathrm{p} .100$ de $\mathrm{DL}$ méthionine ..... & G & - \\
\hline
\end{tabular}

Les levures sont utilisées en substitution de $2 \mathrm{p}$. Ioo de chacune des protéines du régime de base. Ainsi 4 p. roo de protides de levure sont introduits dans les régimes expérimentaux de manière à maintenir constant le taux protidique, soit i4 p. Ioo.

Tous les animaux, Rats et Poussins, sont nourris ad libitum et reçoivent l'eau à volonté. Dans chaque expérience on elf sctue quotidiennem snt le relevé de la mortalité, le contrôle de la consommation d'aliment. Chaque semaine on enregistre le gain de poids individuel des animaux.

\section{RÉSULTATS}

\section{ESSAIS SUR POUSSIN}

Dans les deux expériences qui ont été poursuivies, l'aspect des animaux est satisfaisant. La croissance n'est pas très rapide, mais aucun signe de carence ne se manifeste. Ën particulier l'emplumement est normal. Deux cas de mortalité sont cependant notés sans qu'il soit possible de leur attribuer une cause alimentaire certaine.

$$
\text { Ier essai }
$$

Les résultats portant sur les gains de poids et les consommations d'aliment des animaux qui reçoivent la levıre de bière ou son autolysat en substitution du tourteau d'arachide lorsqu'ils sont incorporés isolément ou en association, figurent dans le tableau 7 .

Annales de Zootechnie. - I902. 
TABLEAU 7

Efficacité comparie de la levure et de son autolysat

\begin{tabular}{|c|c|c|c|c|c|c|}
\hline \multirow[b]{2}{*}{ Régimes } & \multicolumn{3}{|c|}{$\mathrm{f}^{\mathrm{e}}$ semaine } & \multicolumn{3}{|c|}{ ye semaine } \\
\hline & $\begin{array}{l}\text { poids vif } \\
\text { moyen } \\
\text { (g) }\end{array}$ & $\begin{array}{c}\text { Erreur } \\
\left( \pm 2-\frac{\sigma}{\sqrt{\lambda}}\right)\end{array}$ & $\begin{array}{c}\text { Indice de } \\
\text { consommation }\end{array}$ & $\begin{array}{l}\text { poids vif } \\
\text { moven } \\
\text { (g) }\end{array}$ & $\left(\begin{array}{c}\text { Erreur } \\
\left(\approx 2 \frac{\sigma}{\sqrt{x}}\right)\end{array}\right.$ & $\begin{array}{c}\text { Indice de } \\
\text { consommation }\end{array}$ \\
\hline I & $435, \div 1$ & $\pm 32,80$ & $2,1, x$ & 1189,27 & $\quad \ldots 96,58$ & $3,3_{t}^{\prime}$ \\
\hline II & 399,78 & $\pm 20,12$ & $\because, 317$ & 1204,00 & $:=56,60$ & 3,27 \\
\hline III & 439,14 & $\pm 11,2^{\prime}{ }^{\prime}$ & $2.32 \times$ & $1175,36 \mathrm{i}$ & $i \quad \pm 85,60$ & 3,26 \\
\hline IV & 459,50 & $\pm 26,40$ & 2,378 & 1212,23 & $\therefore 87,80$ & $3,3^{\prime}+$ \\
\hline $\begin{array}{l}\text { I - } \\
\text { II }- \\
\text { III } \\
\text { IV }\end{array}$ & $\begin{array}{l}\text { tion de ba } \\
\text { evure sèche } \\
\text { dutolysat d } \\
\text { evure + au }\end{array}$ & $\begin{array}{l}\text { RB } 1 . \\
\text { evure. } \\
\text { ysat. }\end{array}$ & & & & \\
\hline
\end{tabular}

A la quatrième semaine le remplacement isoazoté du tourteau d'arachide par la levure sèche se traduit par une diminution de la croissance de l'ordre de $6 \mathrm{p}$. Ioo. Par contre, les régimes renfermant de l'autolysat (III et IV) manifestent une supériorité hautement significative en ce qui concerne l'intensité de la croissance.

Ces différences ne se manifestent plus à 9 semaines, les gains de poids des différents lots n'étant pas significativement différents. A la $4^{\mathrm{e}}$ semaine. la croissance plus intense des poussins recevant l'autolysat de levure n'a pas pour motif une meilleure utilisation du régime mais une plus grande appétence pour la ration. C'est ce qui ressort du tableau $s$ où sont rassemblées les consommations des différents lots.

TABLEAC 8

Consommation d'aliment par les poussins de' 4 semaines

\begin{tabular}{|c|c|c|}
\hline Lots & $\begin{array}{l}\text { Nature des suppléments du } \\
\text { régime de base }\end{array}$ & $\begin{array}{l}\text { Quantité de nourriture ingérée } \\
(\mathrm{g})\end{array}$ \\
\hline $\mathrm{I}$ & R. $13.1+$ Arachide & 1025 \\
\hline II & R. B. 1 + Levure + Arachide & 940 \\
\hline III & R. B. $1+$ Autolysat + Arachide & 1035 \\
\hline IV & R. B. $1+$ Autolysat + Levure & 1090 \\
\hline
\end{tabular}

La confrontation des résultats de croissance et des taux d'ingestion révèle la même progression des valeurs. Ainsi les gains de poids nettement plus élevés sous l'effet de l'autolysat par rapport à la levure sèche peuvent être attribués uniquement à des taux d'ingestion plus élevés. Cet effet caractérise d'ailleurs tous les produits hydrolysés ou autolysés qui renferment des facteurs d'appétence. 
$2^{\mathrm{e}}$ essai

Les résultats portant sur le gain de poids et l'indice de consommation des poussins recevant la levure entière ou autolysée, supplémenté ou non en méthionine, sont rassemblés dans le tableau 9.

TABLEAU 9

Effet de la supplémentation de la levure par la méthionine selon son mole de préparation

\begin{tabular}{|c|c|c|c|c|c|c|c|c|c|}
\hline \multirow[b]{2}{*}{ Lots } & \multirow[b]{2}{*}{ Nature des Régimes } & \multicolumn{4}{|c|}{3 semaines } & \multicolumn{4}{|c|}{8 semaines } \\
\hline & & $\frac{\text { gain d }}{(\mathrm{g})}$ & $\frac{\text { epoids }}{(\%)}$ & $\begin{array}{l}\text { Nourriture } \\
\text { ingérée }(\mathrm{g})\end{array}$ & I. C. & $\frac{\text { gain d }}{(g)}$ & poids & $\begin{array}{l}\text { Nourriture } \\
\text { ingérée }(\mathrm{g})\end{array}$ & I. C. \\
\hline $\mathrm{V}$ & R. B. I (témoin) & 100 & & 397 & $3,9 \pi$ & 617 & & 2870 & 4,65 \\
\hline VI & R.B. $2+$ lysine + methionine & 137 & 100 & 373 & 2,72 & 755 & 100 & 2906 & 3,84 \\
\hline VII & R. B. 2 + Levure entière & 153 & 112 & 410 & 2,68 & 771 & 102 & 2933 & 3,87 \\
\hline VIII & $\begin{array}{l}\text { R. B. } 2+\text { Levure entière }+ \\
\text { méthionine }\end{array}$ & $16^{\prime} \mathrm{t}$ & 120 & 425 & 2,59 & 839 & 111 & 3081 & 3,67 \\
\hline IX & R. B. 2 + Levure autolysée & 15:3 & 112 & 461 & 3,01 & 766 & $10 \mathrm{~L}$ & 3180 & 4,15 \\
\hline $\mathrm{x}$ & $\begin{array}{l}\text { R. B. } 2+\text { levure autolysée }+ \\
\text { méthionine }\end{array}$ & 178 & 130 & 485 & 2,73 & $8 \% y^{\prime}$ & 809 & $305: 3$ & 3,72 \\
\hline
\end{tabular}

Ils sont comparés à ceux fournis par le régime de base seul supplémenté avec la même quantité de lysine que celle apportée par la levure.

Le régime de base, constitué de protides d'origine végétale de médiocre valeur, manifeste son infériorité malgré la supplémentation en lysine et en méthionine qui correspond aux principales déficiences des céréales et de l'arachide. Rappelons à cet effet qu'un régime classique pour poussin ayant le même pourcentage d'azote mais constitué de céréales et de farine de poisson assure des gains de poids de $20 \mathrm{p}$. cent supérieurs. La levure, sous quelque forme qu'elle soit, valorise le régime, tout au moins pour les premières semaines. Le bénéfice s'atténue au fur et à mesure que l'animal vieillit pour devenir nul à la 8 e semaine.

Lo mode de préparation de la levure n'a ici aucun retentissement sur son efficacité. Les animaux ont le même gain de poids avec les régimes VII et IX à tous les âges. Il en est de même lorsque l'on compare les lots VIII et X à 8 semaines. Le manque d'intérêt de l'autolyse se traduit encore au niveau de l'indice de consommation qui se trouve ainsi déprécié. Le seul fait remarquable est l'avantage que retire la levure de la supplémentation par la méthionine, à la fois pour la croissance et pour l'efficacité du régime. Ce bénéfice demeure tout au long de la croissance. Notons toutefois que, chez les animaux de 3 semaines la levure autolysée est mieux valorisée par l'addition de méthionine que la levure entière.

En ce qui regarde le niveau d'ingestion, les animaux qui reçoivent la levure autolysée ingèrent de grandes quantités d'aliment.

\section{ESSAIS SUR RAT}

Les deux expériences effectuées sur Rat ont porté sur des levures d'origine différentes, levure de bière et levure de lactosérum. Pour la première, nous avons comparé les efficacités de la levure entière, de la levure autolysée et de l'autolysat de levure. 
Pour la seconde, la comparaison a été établie entre la levure sèche et la levure autolysée. Dans tous les cas la supplémentation par o, I p. Ioo de DL méthionine a été étudiée. L'ensemble des résultats figure dans le tableau io.

\section{TABLEAU 10}

Action supplatize de la leante selon son origine, son mode de présentation el son entrichissement en méthionine

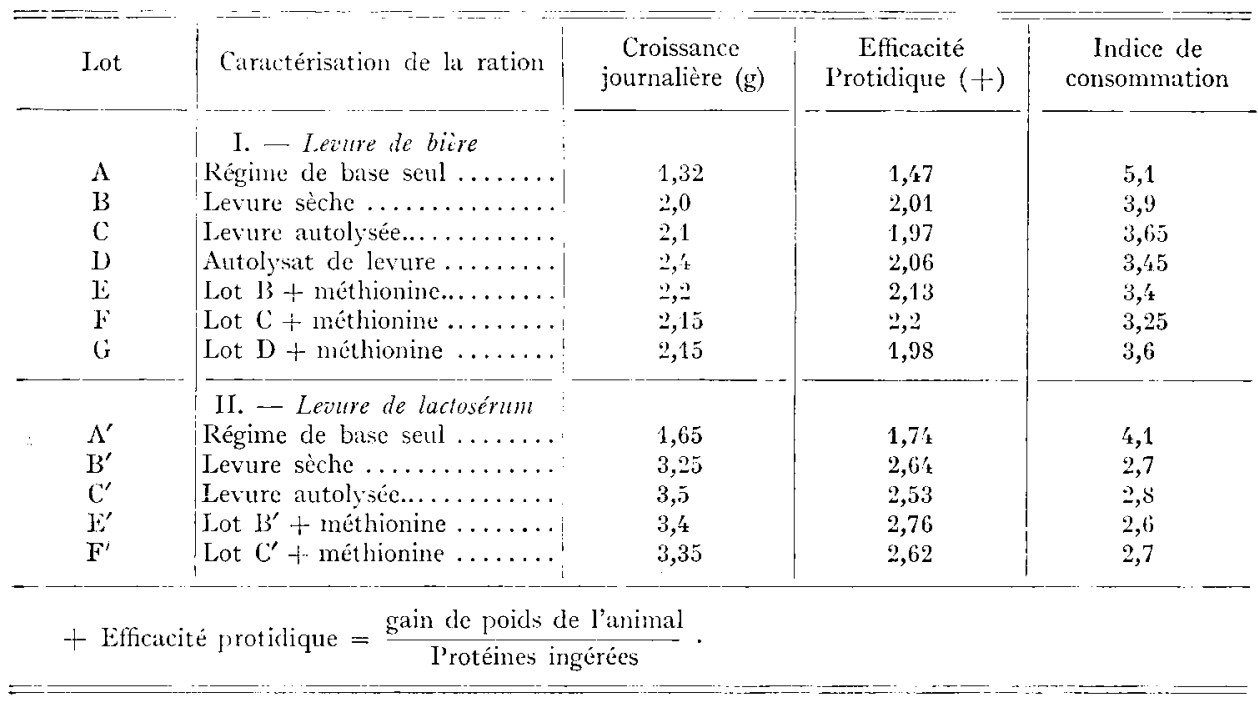

Par rapport à la ration de base, les régimes renfermant de la levure se révèlent nettement supérieurs, tant sur le plan de la croissance qu'au point de vue efficacité protidique. C'est ainsi que pour la levure sèche l'amélioration est la suivante, expri. mée en pourcentage.

Régime de base seul (A ou $A^{\prime}$ )

Croissance protidique

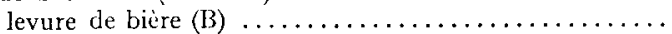

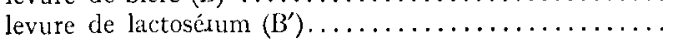

$\begin{array}{ll}- & - \\ 100 & 100 \\ 152 & 137 \\ 198 & 152\end{array}$

Les 2 levures valorisent de façon marquée le régime de base, blé-arachide. D'après sa composition " standard ", la levure de bière supplémente les protides de la ration de base comme il apparait ci-dessous :

Pourcentages de déficit par rapport aux protides totaux de l'œuf :

\begin{tabular}{|c|c|c|c|}
\hline : & $\begin{array}{c}\text { Ration } \\
\text { de base (A) }\end{array}$ & Levure & Ration (B \\
\hline & - & $\rightarrow$ & $\ldots$ \\
\hline Isoleucine $\ldots \ldots \ldots \ldots \ldots \ldots \ldots$ & -59 & -26 & -50 \\
\hline Lysine $\ldots \ldots \ldots \ldots \ldots \ldots$ & -63 & +2 & -42 \\
\hline Méthionine . . . . . . . . . . . & -53 & -54 & -53 \\
\hline Thréonine .................. & -51 & +6 & -31 \\
\hline
\end{tabular}

C'est principalement par sa richesse en lysine que la levure supplémente le régime de base; nous avons, en effet, montré que dans une telle ration dont les protéines sont 
apportées par moitié par l'arachide et par moitié par une céréale, la lysine était le facteur-limitant primaire de ce mélange et que la déficience en méthionine ne constituait ni le facteur limitant primaire, ni le secondaire (ADRIAx et JACQUOT, I96I).

Ceci peut expliquer également que la levure de lactosérum soit plus active que la levure de bière, sa teneur en lysine étant généralement plus élerée (Io p. roo des protides totaux).

Diverses expériences antérieures avaient montré l'intérêt de la levure pour combler le déficit des protides de céréales en 1ysine. JACQUOT et RANDOIN, I942, ont constaté la supériorité de la levure sur l'arachide comme seules sources azotées du régime.

CHICK et SLACK, I945, signalèrent qu'une ration à base de blé était également améliorée par addition de levure. C'est ainsi que 1'efficacité protidique du lot ne recevant que le blé était de 0,94 et qu'elle passait à I,59 pour le mélange blé-levure.

D'après LIGHT et FREY, I943, la levure était aussi efficace que la poudre de lait pour compenser les déficiences de la farine blanche. Enfin, Arckord, I937, a démontré la supériorité de la levure pour supplémenter des régimes végétaux assez voisins de notre ration de base.

La levure sèche valorise donc un régime du type de celui que nous utilisons dans cette expérience.

Si la même levure a subi une autoly'se quelle sera son action supplétive ? Tel est le deuxième point de cette étude.

Des résultats exprimés en pourcentage, dans le tableau I I découlent des principaux faits suivants :

TABLEAU I I

Bénifice apporté par la leiture administrée sous forme autolysic (en $p$. 100 du lot recirant la levure sèche correspondante)

\begin{tabular}{|c|c|c|c|c|c|}
\hline $\begin{array}{l}\text { Forme de } \\
\text { la levure }\end{array}$ & $\begin{array}{l}\text { Type de } \\
\text { levure }\end{array}$ & $\begin{array}{l}\text { Gain de } \\
\text { poids }\end{array}$ & $\begin{array}{c}\text { Consommation de } \\
\text { nourriture }\end{array}$ & $\begin{array}{l}\text { Efficacité } \\
\text { protidique }\end{array}$ & $\begin{array}{c}\text { Indice de } \\
\text { consommation }\end{array}$ \\
\hline Sèche...... & $\begin{array}{c}\text { Bière } \\
\text { Lactosérum }\end{array}$ & 100 & 100 & 100 & 100 \\
\hline & Bière & 105 & 107 & 98 & 99 \\
\hline Autolysée ... . & Lactosérum & 108 & 107 & 96 & $10^{\prime}$ \\
\hline & Moyenne & 100,5 & 107 & 97 & 101,5 \\
\hline Autolysat .... & Bière & $1 \pm 0$ & 116 & 105 & 93,5 \\
\hline
\end{tabular}

- Il existe peu de différence entre l'action de la levure séchée ou autolysée : la croissance et la consommation de nourriture sont légèrement augmentées tandis que l'efficacité protidique a tendance à baisser.

- L'autolysat de levure influence plus nettement le taux de croissance des animaux ainsi que la consommation; ce produit accroît le gain de poids de $20 \mathrm{p}$. cent par rapport à la croissance des animaux recevant la levure sèche.

- Le bénéfice de l'autolysat, et à un titre moindre de la levure autolysée, ne peut être imputé à une meilleure efficacité protidique qui est pratiquement identique dans tous les lots, mais seulement a un effet sur l'appétit des rats ; dans le cas de l'autolysat l'augmentation du taux d'ingestion est de $\mathrm{I} 6 \mathrm{p}$. Ioo et pour la levure autolysée elle est de 7 p. Ioo.

En conclusion, l'autolyse de la levure exerce une action sur l'appétence des 
rations, l'autolysat (concentré sous vide et débarrassé des membranes cellulosiques) étant beaucoup plus actif que la levure autolysée utilisée à l'état brut.

Examinons maintenant l'influence de la méthionine ajoutée aux différentes formes de la levure. Qu'apporte l'extra-méthionine à ces rations ?

Les résultats exprimés en pourcentage des régimes correspondants sans méthionine sont fournis dans le tableau 2.

TABLEAU I 2

Modifications apportées par un suppliment de méthionine aux rations contenant de la levure sous differentes formes (en p. Ioo du lot correspondant sans méthionine)

\begin{tabular}{|c|c|c|c|c|c|c|}
\hline $\begin{array}{l}\text { Forme de } \\
\text { la levure }\end{array}$ & $\begin{array}{c}\text { Type de } \\
\text { levure }\end{array}$ & $!$ & $\begin{array}{l}\text { Gain de } \\
\text { poids }\end{array}$ & $\begin{array}{c}\text { Consommation de } \\
\text { nourriture }\end{array}$ & $\begin{array}{l}\text { Efficacité } \\
\text { protidique }\end{array}$ & $\begin{array}{c}\text { Indice de } \\
\text { consommation }\end{array}$ \\
\hline \multirow{4}{*}{ Sèche.. } & Bière & & +10 & -5 & +6 & -8 \\
\hline & Lactosérum & & $\div 5$ & $\cdots \geq$ & +5 & -4 \\
\hline & Moyenne & & $+7, ;$ & $+3,5$ & $+5,5$ & -6 \\
\hline & Jière & $!$ & +3 & -7 & +12 & -11 \\
\hline \multirow[t]{2}{*}{ Autolyste } & Lactésorum & & $--!$ & -6 & +4 & -4 \\
\hline & Noyenne & & $-0, i$ & $--6, j$ & $\div 8$ & $-7,5$ \\
\hline Autolysat .... & Bière & & -10 & -7 & -4 & +4 \\
\hline
\end{tabular}

- c'est seulement avec la levure sèche que la méthionine améliore légèrement la croissance des animaux (de 7 p. Ioo). Avec les autres formes de levure l'extra-méthionine n'apporte pas de bénéfice sur le développement pondéral des animaux.

- l'efficacité protidique est très légèrement améliorée lorsque la levure sèche ou la levure autolysée est additionnée de méthionine.

- l'efficacité protidique est très légèrement améliorée lorsque la levure sèche ou la levure autolysée est additionnée de méthionine.

- on peut remarquer que dans le cas de l'autolysat de levure l'addition de méthionine présente des répercussions négatives à tous les points de vue, et notamment sur la croissance qui se trouve retardée de Io p. cent en raison d'une diminution de l'ingéré.

- il apparaît (jue la méthionine ajoutée à de la levure ayant subi une autolyse (levure autolysée ou autolysat) diminue la consommation de nourriture d'un taux uniforme de 7 p. I0o. Ce fait, asse\% surprenant demanderait à être confirmé.

lin conclusion, dans nos conditions expérimentales, une addition de méthionine à la levure sèche augmente peu la croissance des a nimaux par suite d'une légère amélioration de l'efficacité protidique. Au contraire, après autolyse de la levure, l'addition de méthionine réduit toujours les ingérés et clans certains cas la croissance s'en ressent.

Un travail mené parallèlement à celui-ci a mis en évidence que le facteur-limitant de la ration de base était la lysine. Ainsi s'expliquent les résultats médiocres obtenus avec l'extra-méthionine qui n'est pas le facteur-limitant des rations utilisées.

\section{DISCUSSION}

La valorisation des régimes végétaux par la levure ressort des résultats de cette étude. Eille est manifeste avec le Rat où la substitution isoazotée de protéines de levure aux protéines d'arachide et au gluten augmente la croissance de $50 \mathrm{p}$. Ioo dans le cas 
de la levure de bière et de Ioo $\mathrm{p}$. Ioo dans le cas de la levure de lactosérum. Cet effet n'est pas toujours aussi marqué chez le Poulet. Au cours du second essai où la totalité dı gluten était remplacé par un apport isoazoté de levure on observe un accroissement de $50 \mathrm{p}$. Ioo du taux de croissance mais il n'en est pas ainsi au cours du premier essai où la substitution de la levure n'a pas d'incidence favorable sur le gain de poids de l'animal.

Par rapport à la levure sèche, la levure autolysée utilisée sous forme entière ne prèsente aucune supériorité quant à la croissance du Poulet et à son indice de consommation. Le Rat réagit plus favorablement mais les très faibles différences de croissance (6 p. Ioo) vont de pair avec un niveau d'ingestion légèrement plus élevé ( $7 \mathrm{p}$. IOo).

L'autolysat possède un effet très net sur la croissance. Par rapport à la levure sèche, le bénéfice qu'entraîne son emploi est d'environ ro p. Ioo avec le Poulet et 20 p. Ioo avec le Rat. Cette supériorité de l'autolysat n'est qu'apparente. En effet chez le Rat, les indices de consommation et les coefficients d'efficacité protidique sont du même ordre de grandeur avec la levure sèche et son autolysat. Ils sont strictement identiques dans le cas du Poulet. Le bénéfice imputable à l'autolysat est essentiellement le fait d'un taux d'ingestion plus élevé. Chez le Rat et le Poulet, il existe une relation étroite entre les gains pondéraux et les quantités de nourriture consommées.

En soi, l'autolyse n'exerce aucune influence sur la disponibilité des aminoacides de la levure comme le montre 1'identité de réponse des lots placés à la levure sèche et à la levure autolysée. L'effet d'appétence caractérise l'autolysat de levure sous sa forme entièrement soluble et débarrassée de toute cellulose. On peut 1'attribuer à la présence d'acides aminés libres puisque l'un de nous a montré qu'il en était ainsi lorsque l'on ajoute des aminoacides purs à la ration du. Poulet. Or, au cours de l'autolyse l'azote protéique de la levure est transformé en azote aminé comme l'indique le tableatr I puisque l'azote de l'autolysat ne titre plus au sulfate de cuivre. Cependant on comprend mal dans ces conditions pourquoi il n'en est pas également ainsi dans le cas de la levure autolysée qui n'agit pas sur l'appétit des animaux à la manière de l'autolysat.

Dans la pratique, l'emploi d'autolysats solubles de levure peut être pris en considération comme facteur d'appétence, retentissant nécessairement sur la vitesse de croissance. On sait d'ailleurs que la majorité des autolysats, protéolysats, hydrolysats ont une influence analogue quelle qu'en soit 1'origine (poisson, viande, gruyère).

Chez le Poulet, la levure est utilement supplémentée par adjonction de méthionine. A l'âge de 3 semaines c'est après autolyse que la levure se prête le mieux à cette supplémentation ; mais à 8 semaines on observe la même valorisation quelle que soit la forme de levure utilisée. Chez le Rat, l'extra-méthionine n'a pratiquement pas eu d'effet sur la croissance et sur le coefficient d'efficacité protidique. Il est presque certain que cette incapacité est le fait de la nature même des régimes utilisés qui avaient la lysine comme facteur limitant primaire.

Les résultats de cette étude portent à penser qu'une dessiccation suffisamment intense pour tuer tous les microorganismes, arrêter les fermentations et dilacérer les membranes cellulosiques des cellules suffit à conférer le caractère disponible aux constituants de la levure. Nos conclusions sont à rapprocher de celles auxquelles avait abouti un travail antérieur sur les farines et les autolysats de poisson (FÉvRIER et al., I954). 


\section{SUMMARY}

\section{STUDY ON THE NUTRITIONAL VALUE OF THREE DIFFERENT YEAST PREPARATIONS : DRIED YEAST, AUTOLYZED YEAST AND YEAST-AUTOLYSATE}

The object of the present study is a comparison of the growth efficiency of three different forms of yeasts : complete dried-yeast, autolyzed yeast and yeast autolysate.

The first sample is dehydrated on cylinders at $13 \mathrm{o}^{\circ} \mathrm{C}$. The two others are prepared from dried yeast subjected to autodigestion. Yeast is maintained under adequate conditions of heat $\left(50^{\circ} \mathrm{C}\right), \mathrm{pH}$ (5) and asepsis during the 48 hour autolysis. After drying the product, autolyzed yeast is obtained. This substance contains all the yeast constituents, including cellulose of the cytoplasmic membranes. Veast autolysate is produced by high-speed centrifugation of the autolyzed yeast, a process which separates the cellulosic membrane from the soluble fraction. The latter is then dried to give the yeast autolysate, a pasty product still containing $30 \mathrm{p}$. I0O water. An analysis of the samples is presented in table I.

The three different forms of yeast - prepared also from brewer's yeast (Saccharomyces cerevisiae) and from yeast grown on Saccharomyces lactis -- were studied in the rat and the chick. Diets with and without supplementary methionine were compared. The extent to which autolysis increases the availability of yeast nutrients, especially that of methionine, was investigated.

From our results it can bee seen that ;

$\mathrm{I}^{\mathrm{O}}$ The two types of yeast are capable of increasing the value of a diet consisting of vegetable protein (peanut, gluten) when isonitrogenous substitution is made.

$2^{\circ}$ Yeast autolysate yieldeld faster growth than untreated yeast.

$3^{\circ}$ This improvement, however, was only apparent since it was essentially manifested by increased intakes. In both cases, feed and protein efficiencies were strictly the same. The autolysate is interesting as a factor improving palatability.

$4^{\circ}$ With a lysine deficient diet (in the case of rats) addition of methionine to the yeast resulted in no improvement whatever ther treatment or the origin of the yeast used. With a well balanced diet (in the case of chicks) yeast is improved by methionine supplementation, but the improvement is the same for the three different yeast preparations.

It appears that methionine is equally available in dried and autolyzed yeast, and that simple drying on cylinders is sufficient to allow complete utilisation of this amino acid.

\section{RÉFÉRENCES BIBLIOGRAPHIQUES}

ADRIAN J., JACQUOT R., ig6r. Essais comparés de supplémentation d'une ration à base de sorgho et d'arachide par la farine de poisson ou par des acides aminés purs. Ann. Nutrit. Alim., 16, 227-237.

Aykroid W. R., 1937, in JacQuor. 1946. Cité plus loin.

Baldini J. T., RosenbERG H. R., I955. The effect of productive energy level of the diet on the methionine requirement of chick. Poul. Sci, 34, I 30 I-I 307 .

CHICK H., SLACK E.-B. I945. Note on the nutritive value of the nitrogenous substances contained in dried yeast (Torulopsis Lipofera). Biochem. J., 39, 164-167.

Fevrier R., Jacquot R., Matet..J., Pero R., i954. Influence du mode de préparation sur la valeur nutritive de trois farines de poissons. Ant. Zootech., 3, 223.246.

FRAPS G. S., I946. Composition and productive energy of poultry feeds and rations. Texas. Agric. Exp. Sta. Bull. $n^{0} 6^{6} 78$.

JACQUOT R., RANDON L., I942, Etude biologique de la valeur nutritive comparée de la farine de tourteaux d'arachide déshuilée et des levures sèches, avec le jeune Rat comme réactif animal. Bull. Soc. Chim. Biol., 24, $165-167$.

JACQvot R., 1946. Valeur alimentaire de quelques protides de remplacement. Bull.Soc. Sci. Hyg. Aliment., 33, 799-805.

Light R. F., Frey C. N., I943. The nutritive value of white and whole wheat breads. Cereal. Chem. 20, $645-660$. 